ISSN: 1130-3743 - e-ISSN: 2386-5660

DOI: http://dx.doi.org/10.14201/teoredu2017291189216

\title{
LA IDEA VECTOR Y SUS ESFERAS: UNA PROPUESTA FORMATIVA PARA LA AMBIENTALIZACIÓN CURRICULAR DESDE LA COMPLEJIDAD ${ }^{1}$
}

\section{The Vector Idea and its spheres: a training proposal for curriculum greening processes from the complexity}

\section{L'Idée Vecteur et ses sphères: une proposition de formation pour l'écologisation des programmes d'études du point de vue de la complexité}

Genina CALAFELL y Mercè JunYeNT

Universitat Autònoma de Barcelona. Departamento de Didáctica de la Matemática y las Ciencias Experimentales.genina.calafell@uab.cat; merce.junyent@uab.cat

Fecha de recepción: enero de 2017

Fecha de aceptación: marzo de 2017

RESUMEN

El estudio se sitúa en el marco de la Educación para la Sostenibilidad y, concretamente, en los procesos de Ambientalización Curricular. En primer lugar, se expone brevemente una conceptualización acerca de la Ambientalización Curricular siguiendo el modelo ACES (Ambientalización Curricular de los Estudios Superiores). A continuación, se presenta la investigación que se concreta en el análisis del grado

1. Investigación realizada en el marco de MCYT:EDU2009-13893-CO2-01EDUC/AGAUR (Conselleria d'Innovació, Universitats i Empreses): 2014SGR543/Departament de Territori i Sostenibilitat de la Generalitat de Catalunya. 
de Ambientalización Curricular de un programa formativo dirigido a profesionales de la educación. Este ha sido elaborado desde la perspectiva de la complejidad y se basa en la propuesta de Idea Vector y sus esferas (conceptual, metodológica, creativa, de investigación). Se trata de una investigación interpretativa, siendo los participantes los profesores que siguieron la formación. Los resultados permiten obtener orientaciones para el desarrollo de un proceso formativo en Ambientalización Curricular, coherente con el modelo ACES, y con impacto real en los participantes. Estas orientaciones se fundamentan en las ideas siguientes: credibilidad, cosmovisión, conceptualización y acción.

Palabras clave: Educación para la Sostenibilidad; Ambientalización Curricular; complejidad; programa formativo; formación del profesorado; Idea Vector.

\section{SUMMARY}

The study is in the framework of Education for Sustainability and, specifically, in the curriculum greening processes. In the first place, a conceptualization about curriculum greening is briefly presented following the ACES model. In the second instance, the research is exposed. It is concretized in the analysis of the degree of curriculum greening in a training program aimed at education professionals. This training programme has been elaborated from the perspective of complexity and based on the proposal of Vector Idea and its spheres (conceptual, methodological, creative, and investigative). The research carried out has an interpretative approach and the participants were teachers who followed the training programme. The results allow obtain guidelines for the development of a training process in curriculum greening, coherent with the ACES model, and with real impact on the participants. These orientations are based on the following ideas: credibility, cosmovision, conceptualization and action.

Key words: Education for Sustainability; curriculum greening; complexity; training programme; vector idea; teacher training.

\section{SOMMAIRE}

L'étude s'inscrit dans le cadre de l'éducation pour le développement durable et plus particulièrement dans l'écologisation des programmes d'études. En premier lieu, nous exposons brièvement une conceptualisation autour de l'écologisation des programmes d'études, selon le modèle ACES. Nous présentons ensuite la recherche, qui porte sur l'analyse du degré d'écologisation d'un programme de formation destiné aux professionnels de l'éducation. Ce programme a été élaboré à partir de la perspective de la complexité et il est fondé sur la proposition d'Idée Vecteur et de ses sphères (conceptuelle, méthodologique, créative, de recherche). Il s'agit d'une recherche interprétative, les participants étant les professeurs ayant reçu la formation. Les résultats permettent d'obtenir des orientations pour le développement d'un processus de formation en écologisation des programmes d'études, cohérent avec le 
modèle ACES, et avec un impact réel sur les participants. Ces orientations se fondent sur les idées suivantes: crédibilité, cosmovision, conceptualisation et action.

Mots clés: Éducation pour le développement durable; écologisation des programmes d'études; programme de formation; formation des enseignants.

\section{INTRODUCCIÓN}

El estudio que se presenta se sitúa en el marco de la Educación para la Sostenibilidad que se dirige a la transformación, asumiendo que una educación transformadora conlleva ofrecer nuevas formas de ver el mundo, supone el desarrollo de un pensamiento holístico, sistémico, crítico y creativo, y el fortalecimiento del empoderamiento de la ciudadanía para una toma de decisiones informada, responsable y comprometida en la construcción de escenarios futuros alternativos. La investigación pretende identificar los factores significativos de un programa formativo de Ambientalización Curricular y proponer orientaciones de continuidad y mejora del programa. Para ello se fundamenta en trabajos realizados en el marco del Grupo de Investigación Complex (Bonil et al., 2012; Canelo, Junyent y Bonil, 2015; Calafell, Junyent y Bonil, 2015).

\section{PROCESOS DE AMBIENTALIZACIÓN}

De acuerdo con el informe GuNi (2012), la introducción de la sostenibilidad en la educación está siendo lenta en relación a la urgencia de la situación y los cambios necesarios. Si la educación quiere ser transformadora, se debe considerar que es sujeto y agente de cambio, por lo que también tiene que transformarse y de manera profunda. No se trata de introducir nuevas asignaturas ni actividades puntuales en algunas de las ya existentes, sino que se deben afrontar y llevar a cabo cambios importantes en la gestión de las instituciones educativas, en los procesos de enseñanza-aprendizaje, en cómo se vinculan los centros educativos con la comunidad, y todo ello teniendo en cuenta la monitorización y evaluación de estos cambios (Calafell, Junyent y Bonil, 2015).

La integración de una dimensión ambiental y de sostenibilidad, desde una perspectiva integral de las instituciones educativas es ya un reto ineludible para nuestra sociedad. Esta integración la denominamos ambientalización, que de acuerdo con Pujol (2000) se puede definir como el conjunto de acciones de características diversas que se dirigen a la introducción de la sostenibilidad mediante la reflexión sobre el modelo de crecimiento de nuestra sociedad y sus consecuencias, y la necesidad de nuevos valores sociales que hacen posible un modelo de desarrollo sostenible. En el proceso de ambientalización de una institución educativa convergen al menos tres ámbitos: el estructural, el ciudadano y el curricular.

El ámbito estructural hace referencia a las decisiones de carácter organizativo del centro educativo: secretaría, administración, gestión de recursos y residuos... 
por lo que introducir la ambientalización en este ámbito implica decisiones como, por ejemplo, realizar las fotocopias a doble cara, adquirir el tipo de papel que se utiliza con criterios ambientales, dotar al centro de expendedores de agua de bajo consumo y de contenedores de recogida selectiva de residuos e incluso dotarse de una normativa de uso de las instalaciones.

El ámbito ciudadano hace referencia a la manera en que la comunidad que forma el centro educativo participa y percibe el proceso de ambientalización. Introducir la sostenibilidad en este ámbito requiere dotarse de modelos de organización de las personas y procesos de toma de decisiones de carácter democrático. Algunas experiencias en el ámbito estructural son las comisiones de sostenibilidad de los centros educativos, la introducción de una responsabilidad específica sobre ambientalización en los órganos de gobierno del centro, la creación de espacios de participación de todos los agentes que forman el centro: familias, alumnado, profesorado, personal de administración y servicios.

El ámbito curricular, el de Ambientalización Curricular (Junyent, Geli y Arbat, 2003), en algunos ámbitos denominado Sostenibilización Curricular (CADEP, 2005), se orienta hacia la forma en que la educación para la sostenibilidad se introduce en el currículum del centro educativo. En este sentido, la literatura nos muestra diversidad de modalidades. Está muy extendida la introducción de la educación para la sostenibilidad en una asignatura, generalmente las ciencias naturales, a partir de un centro de interés o al final de un tema; o bien, la realización de semanas culturales donde el centro educativo trabaja el currículum de forma interdisciplinaria tomando como centro de interés un tema específico, por ejemplo la semana del agua; o modelos donde la educación para la sostenibilidad tiene presencia en las tutorías al debatir con el alumnado ciertos temas de controversias socioambientales. Estas opciones no pueden ser consideradas procesos de ambientalización curricular, ya que éstos deben ir más allá de cápsulas curriculares, a menudo desconectadas de las secuencias de aprendizaje que se trabajan en el aula y de la gestión ambiental del centro, si se pretende una incorporación profunda y efectiva en relación a la formación de ciudadanos responsables, críticos y comprometidos con la acción para la transformación hacia una sociedad más justa, equitativa y sostenible. Es en este ámbito, el de la Ambientalización Curricular, donde centramos el estudio.

\section{AMBIENTALIZACIÓN CURRICULAR}

En el presente estudio entendemos la Ambientalización Curricular como «un proceso continuo de producción cultural tendiente a la formación de profesionales comprometidos con la búsqueda permanente de las mejores relaciones posibles entre la sociedad y la naturaleza, atendiendo a los valores de la justicia, la solidaridad y la equidad, aplicando los principios éticos universalmente reconocidos y el respeto a las diversidades» (Junyent, Geli y Arbat, 2003, 21). Un reto fundamental en los procesos de Ambientalización Curricular es el cambio de la cosmovisión que tienen los individuos sobre el mundo (Tilbury, Podger y Reid, 2004; Sauvé, 2006) y 
su consecuente cambio en las maneras de pensar y actuar. En este sentido apostamos por un currículum en el que independientemente de los contenidos tratados y la estructura en que se organiza, la Ambientalización Curricular tiene una presencia permanente, aunque dinámica y contextualizada en cada situación educativa.

Tomamos como referente para orientar los procesos de Ambientalización Curricular en los centros educativos la propuesta de características e indicadores de evaluación del grado de Ambientalización Curricular que propone la Red ACES (Ambientalización Curricular de los Estudios Superiores), propuesta denominada modelo ACES (Geli, Junyent y Arbat, 2003) (Fig. 1).

\section{FIGURA 1}

MODELO ACES, INDICADORES DE EVALUACIÓN DEL GRADO DE AMBIENTALIZACIÓN CURRICULAR DE ESTUDIOS SUPERIORES

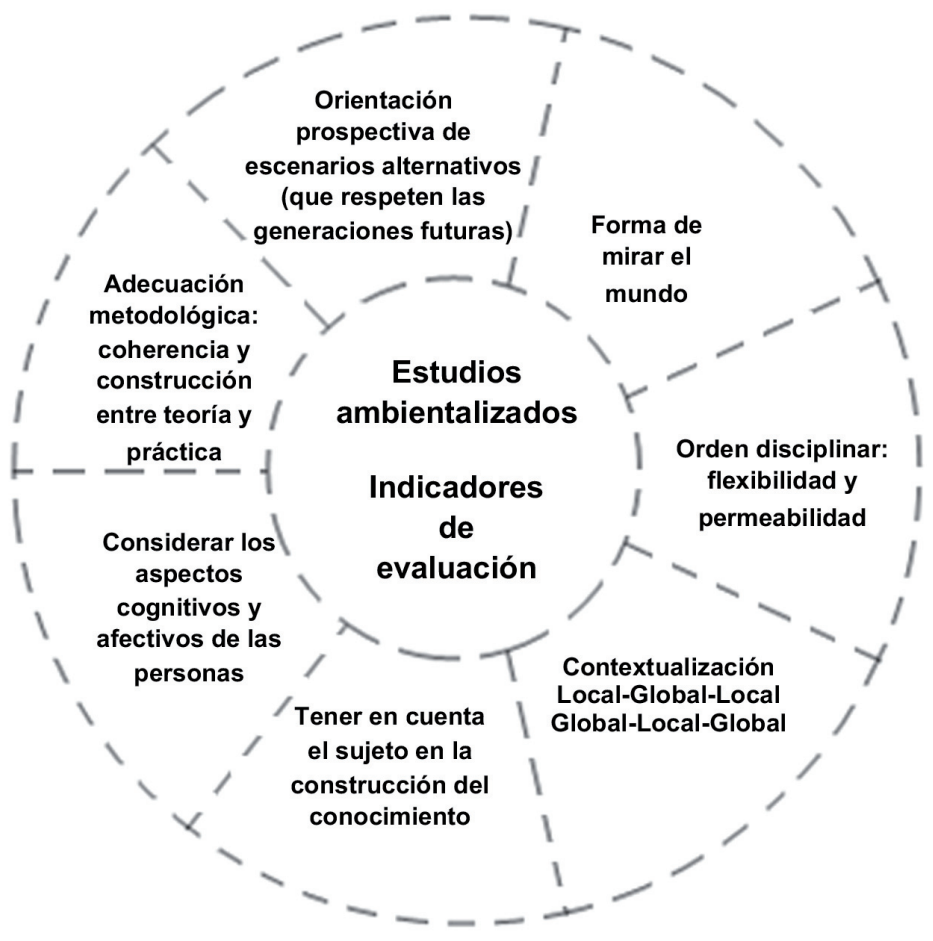

El modelo ACES responde a una visión de la Ambientalización Curricular como sistema complejo formado por siete categorías, las cuales no responden a una ordenación jerárquica y sí a un proceso continuo de cambio orientado a la mejora educativa. Cada una de estas categorías se interpreta de la siguiente manera: 
- Forma de mirar el mundo: integra principios de la complejidad para interpretar los fenómenos del mundo, siendo estos principios el sistémico, el hologramático y el dialógico. Es una visión que asume la multicausalidad, la retroacción, el azar, la irreversibilidad, el dinamismo, el diálogo entre diferentes niveles de escala y la autoorganización en la aproximación a los fenómenos del mundo. El conocimiento es considerado como un proceso continuo, en el cual la duda es un elemento generador del mismo y la creatividad tiene una gran relevancia.

- Orden disciplinario: flexibilidad y permeabilidad. Supone entender que las disciplinas se permeabilizan entre ellas, que admiten una nueva manera de relacionarse, sin perder su identidad, desde una reflexión crítica. Implica la participación de diferentes profesionales, de diversas áreas de conocimiento y la reflexión de cada disciplina para asumir tanto sus límites en la aproximación a los fenómenos del mundo como los retos que esta aproximación genera. Se considera relevante la incorporación de las emociones y la capacidad para una toma de decisiones informada, responsable y comprometida.

- Contextualización: establecimiento de relaciones entre la/s disciplina/s y el entorno, inmediato y global. Consideración de la multidimensionalidad del medio y aproximación a los fenómenos del mundo desde esta perspectiva, desde contextos locales y globales, desde los aspectos cognitivos y afectivos de las personas, desde diversidad de visiones y dimensiones culturales que conviven en nuestra sociedad.

- Tener en cuenta el sujeto en la construcción del conocimiento: la participación activa del alumnado en el proceso de enseñanza-aprendizaje es esencial. Esta participación deberá regularse en todos los ámbitos: organización curricular, gestión del aula, evaluación, planes tutoriales... La atención a la diversidad debe estar especialmente considerada.

- Considerar los aspectos cognitivos y afectivos de las personas: supone dar apoyo al alumnado integrando diferentes niveles: cognitivo, pedagógico y emocional. Se deben trabajar las capacidades de análisis, contextualización y globalización en relación a habilidades sociales como trabajo cooperativo, comprensión y empatía; al mismo tiempo que se incorporan actividades que permitan interpretar la realidad desde la toma de conciencia del pensamiento sistémico y de la sensibilidad y la creatividad.

- Adecuación metodológica: la metodología educativa aplicada relaciona la teoría y la práctica, permitiendo una continua reconstrucción entre ellas. Parte de las ideas, conocimientos, sensaciones, sentimientos y actitudes del alumnado y favorece dinámicas participativas de reflexión y acción democráticas. En esta perspectiva toman relevancia los programas de acción e intervención en la comunidad, cercana y/o global, y en el medio en general. 
- Orientación prospectiva de escenarios alternativos: incorporación de la reflexión en relación a una dimensión de futuros alternativos. Ello supone relacionar perspectivas individuales y colectivas en la proyección de escenarios futuros, así como el análisis crítico del conocimiento tecnocientífico en la sociedad para formar ciudadanos comprometidos y responsables en la consecución de una transformación social hacia futuro más sostenible.

Avanzar hacia un currículum que contemple estas categorías implica pensar, diseñar, implementar y evaluar propuestas formativas que contribuyan al desarrollo profesional de los maestros y profesores en procesos de Ambientalización Curricular.

\section{Propuesta formativa en Ambientalización CurRicular: MOdelo IdeA Vector}

Una propuesta formativa para avanzar en la Ambientalización Curricular desde la complejidad (Calafell, Junyent y Bonil, 2015) se fundamenta en tres principios esenciales:

- La transformación del entorno desde la incorporación de la relación medio y sociedad para la sostenibilidad.

- El desarrollo del pensamiento crítico y creativo de los alumnos desde un diálogo entre ser profesional y ser ciudadano.

- El empoderamiento de los alumnos, los profesores y las instituciones.

Considerando estos principios y tomando como referente el modelo ACEs, el Grupo de Investigación Cómplex de la Universitat Autònoma de Barcelona aporta una propuesta formativa fundamentada en un sistema complejo que toma relevancia tanto en sus componentes como en las relaciones que se establecen entre ellos. Esta propuesta se fundamenta en una Idea Vector, que es motor de la actividad, y las esferas conceptual, metodológica, creativa y de investigación, que la concretan en el escenario del aula. Todo ello considerando que la relación tiempo-espaciocontexto enmarca la acción de Ambientalización Curricular y se concibe como una propuesta abierta a cambios: dinámica y en evolución (Fig. 2). A continuación, se detalla la propuesta y sus componentes. 
FigURA 2

MODELO FORMATIVO ORIENTADOR DE LA FORMACIÓN HACIA LA AMBIENTALIZACIÓN CURRICULAR

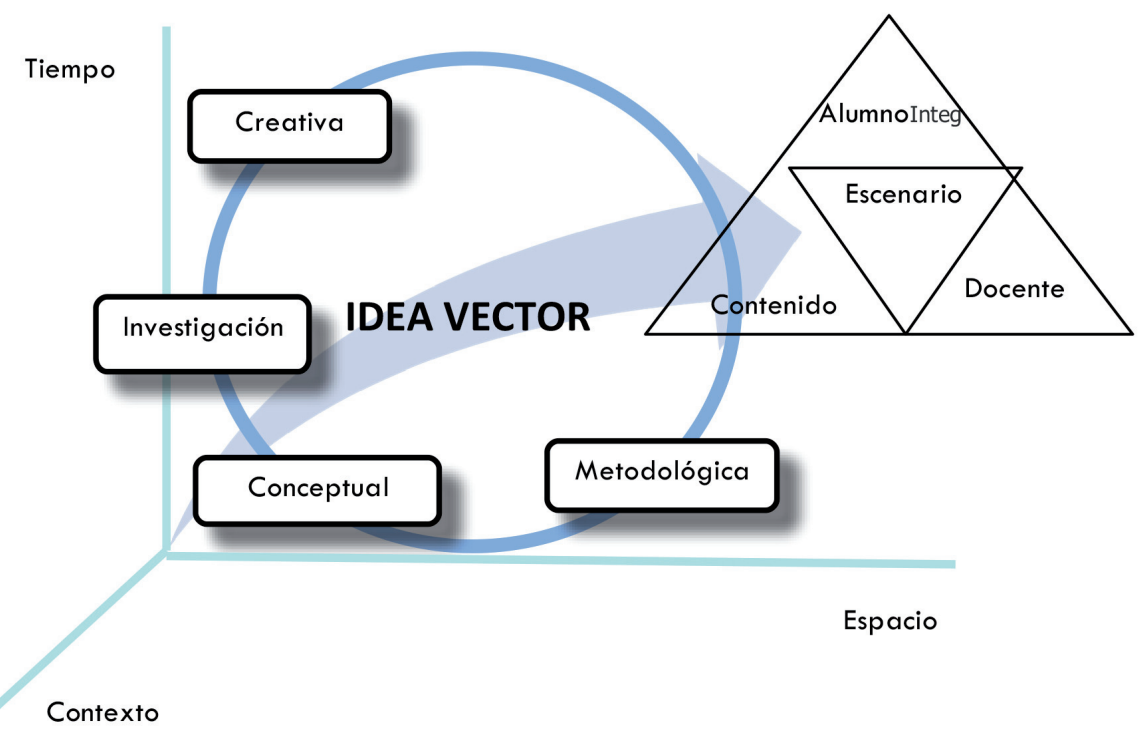

\subsection{La relación tiempo-espacio-contexto}

Nos parece significativo asumir que toda experiencia educativa se ubica de forma simultánea en los ejes espacio, tiempo y contexto sociocultural. Ello determina que para entender una acción educativa es necesario tener en cuenta dónde se desarrolla, cuándo se desarrolla y qué significado tiene para sus participantes. De la interacción entre tiempo, espacio y contexto se construye y se comparte, entre los participantes y el educador de la acción didáctica, una forma de entender, ser y actuar en el mundo. Es decir, se dibuja una cosmovisión particular que da singularidad a la actividad que genera y que hace que sea difícil repetir la misma experiencia de forma mecánica y seriada. Una experiencia que ha de favorecer que los individuos reflexionen sobre su cosmovisión y tengan elementos para decidir si la cambian; en este sentido la educación para la sostenibilidad se convierte en una educación con potencial para revisar la propia cosmovisión (Sauvé, 2006).

\subsection{La Idea Vector}

El modelo educativo que se presenta propone que de la relación entre tiempo, espacio y contexto emerge una idea principal que puede focalizar la acción 
educativa. Esta idea principal la denominamos Idea Vector (Fig. 2), la cual orienta y vertebra toda la acción educativa y es una manera de aproximarse al mundo desde la transformación. Metafóricamente, podrían ser las gafas que ponemos a los participantes de la acción educativa para que sean capaces de ver en su entorno nuevas entidades y relaciones. La Idea Vector es transversal a las áreas de conocimiento, incorpora la perspectiva de la complejidad y tiene un fuerte componente social. La mirada desde la complejidad aporta entender el mundo desde el principio dialógico (entidades aparentemente contrarias pueden dialogar y ser complementarias), el principio sistémico (el todo es más que la suma de las partes) y el principio hologramático (un sistema sigue unas reglas escalares entre lo macro y lo micro en forma de holograma o fractal).

Para pensar y seleccionar una Idea Vector hay que ir más allá de conceptos curriculares y evitar valores éticos para no caer en contenidos o moralejas preestablecidas o dogmáticas. A la vez, la experiencia profesional y personal del equipo que diseña y ejecuta un programa formativo es esencial ya que condiciona la opción final de una Idea Vectora. Sin la intención de dar listas cerradas de ideas vector, Inimaginable, Contraintuitivo, Dinamismo, Perspectiva o Controversia son ejemplos de ideas vector utilizadas en acciones de ambientalización del currículum. Las ideas vector tienen, además, como particularidad un carácter nómada y mutante. ¿Qué queremos decir con esto? Para explicarlo vamos a utilizar la metáfora de una jam session:

En el encuentro entre los músicos de jazz no hay ninguna partitura escrita o ensayada y son las reglas culturales de la propia jam session las que facilitan a los intérpretes improvisar con rigurosidad y creatividad, individualidad y colectividad. La misma jam session evoluciona a partir de la interacción de los músicos y la interpretación que se va (re)construyendo. De hecho es una efervescencia musical que es nómada, en la medida que aparece y desaparece de un espaciotiempo-contexto, pero deja un saber compartido entre los participantes (músicos y público). A la vez es mutante porque es un encuentro permanentemente dinámico y abierto en el que el punto de partida de una interpretación puede dar a lugar a multitud de hilos musicales continuos. Además, el punto final de una interpretación puede ser el inicio de otra (Calafell, Junyent y Bonil, 2015, 58).

\subsection{Las esferas: conceptual, metodológica, creativa, de investigación}

El modelo formativo propuesto define cuatro esferas: la esfera conceptual, la esfera metodológica, la esfera creativa y la esfera de investigación. Las esferas ayudan a concretar la Idea Vector en el escenario didáctico y permiten construir la idea vector en la acción educativa considerando los contenidos a enseñar y aprender, el rol del profesor y el protagonismo del alumnado.

Estas esferas están íntimamente relacionadas entre ellas a modo de engranaje y se retroalimentan entre sí, de modo que en su concreción, cuando se opta por una idea en una esfera, ésta influye en la construcción de las otras. 
La esfera conceptual conecta la idea vector con los contenidos curriculares de la acción formativa. Debe tener un claro y compartido significado para el colectivo al que se dirige la acción educativa y permitir estimular la reflexión y la acción en torno al mundo y la cotidianidad de los participantes. Esto implica que, para una misma Idea Vector, podemos definir, únicamente tomando como referencia si el alumnado es de primero de primaria o de tercero de secundaria, esferas conceptuales distintas.

La esfera creativa aporta una visión cultural a la acción didáctica desde una perspectiva estética, artística y transformadora. Esta dimensión implica que se considera una perspectiva divergente (o aparentemente no tradicional) en la comprensión del mundo y de su relación con los contenidos que se trabajan. La dimensión creativa implica favorecer la indagación -tanto individual como colectiva- de relaciones a priori antagónicas como emoción y razón, objetividad y subjetividad $\mathrm{o}$ arte y ciencia. Una reflexión creativa que invita a los participantes a comprender el mundo desde una mirada dialogante entre distintas perspectivas, a veces aparentemente inconexas.

La esfera metodológica lleva a la reflexión sobre las opciones de cómo se realizará la acción formativa y qué estrategias didácticas se utilizarán en la actividad, manteniendo una coherencia y pertinencia con la idea vector y las esferas conceptual y creativa. Esta dimensión implica decisiones en relación a la dinámica de aula, los instrumentos didácticos, la organización de los participantes, el rol del profesorado, la temporización y secuenciación de la acción o la forma de evaluar.

La esfera de investigación abre la puerta a una visión de los escenarios educativos como oportunidades para estimular la investigación. Pretende favorecer que los participantes comprendan la acción educativa como una oportunidad para abordar la práctica reflexiva rigurosa y el contraste de diversos puntos de vista. Si el objetivo de la práctica educativa no considera la investigación esta esfera no se concretaría.

La Idea Vector y las esferas se proyectan sobre la actividad docente y ésta es entendida como un escenario. La palabra escenario toma su significado etimológico: skené, donde el escenario es el espacio donde los actores representan la obra a partir de la combinación entre skené y graphos que da lugar a la skenographia, el conjunto de complementos que hacen posible la representación de la obra. La actividad educativa se convierte, por lo tanto, en una experiencia creada por sus actores, donde docentes, alumnado y currículum se encuentran en una construcción efímera modulada por cuatro esferas que conectan el conjunto con una gran finalidad en términos de cambio de cosmovisión. El aula se convierte en un espacio constructor y construido, un espacio de diálogo entre agentes de gran fuerza comunicativa en que sobre todo se espera que "pasen cosas" en el sentido más amplio de la expresión. Espacio donde se opta por un currículum que dialoga entre la especificidad de las áreas de conocimiento, sus modelos explicativos y los fenómenos del mundo sobre los que proyecta su conocimiento la ciudadanía. 


\section{OBjetivos}

La investigación pretende analizar el grado de Ambientalización Curricular de un programa formativo orientado a profesionales de la educación. Esta finalidad constituye una oportunidad para avanzar en la investigación de los procesos de Ambientalización Curricular y para ello se plantean los siguientes objetivos y preguntas de investigación:

1. Identificar los factores del programa formativo que son significativos en relación a la Ambientalización Curricular. Para ello nos preguntamos: ¿Qué categorías de la ambientalización curricular destacan los participantes en el programa formativo? ¿Qué factores del programa se destacan como oportunidades? ¿Qué factores del programa se destacan como obstáculos?

2. Proponer orientaciones de continuidad del programa formativo en relación a la mejora de la Ambientalización Curricular. Para ello nos preguntamos: ¿Qué orientaciones de continuidad se derivan de los resultados de la investigación?

\section{Metodología}

\subsection{Contexto y participantes}

La investigación que se presenta se contextualiza en una formación realizada a profesores, educadores, asesores y técnicos de la XESC (Red de Escuelas Verdes de Catalunya) que impulsa el gobierno autonómico (Departament d'Ensenyament y Departament de Territori i Sostenibilitat de la Generalitat de Catalunya) y que lidera el Grupo de Investigación Cómplex.

El programa formativo pretende impulsar y promover la incorporación de la educación para la sostenibilidad en los centros educativos y apuesta por avanzar en la ambientalización del currículum desde un enfoque innovador y fundamentado en la investigación en complejidad.

El programa se diseña y se ejecuta tomando como referencia el modelo formativo de Idea Vector expuesto y se estructura en seis sesiones formativas de 8 horas de duración. En la Tabla 1 se expone la programación siguiendo el modelo formativo referenciado. 
LA IDEA VECTOR Y SUS ESFERAS: UNA PROPUESTA FORMATIVA PARA LA AMBIENTALIZACIÓN CURRICULAR...

\begin{tabular}{|c|c|c|c|c|}
\hline $\begin{array}{c}\text { Tí́tUlo } \\
\text { DE LA SESIÓN }\end{array}$ & IDEA VECTOR & $\begin{array}{c}\text { ESFERA } \\
\text { CONCEPTUAL } \\
\end{array}$ & $\begin{array}{c}\text { ESFERA } \\
\text { DIDÁCTICA } \\
\end{array}$ & $\begin{array}{c}\text { ESFERA } \\
\text { CREATIVA }\end{array}$ \\
\hline Transparencias & Inimaginable & Techo de cristal & Representar & Instalaciones \\
\hline $\begin{array}{c}\text { De lo inimaginable } \\
\text { a lo intangible }\end{array}$ & Intangible & Azar & Juegos de azar & Pintura \\
\hline In \& out & Optimismo & $\begin{array}{c}\text { Estrategia de } \\
\text { cambio }\end{array}$ & $\begin{array}{c}\text { Construir para } \\
\text { transformar }\end{array}$ & Poesía visual \\
\hline Sinapsis ambiental & Conectar & $\begin{array}{l}\text { Competencias } \\
\text { profesionales }\end{array}$ & Crear & Jazz \\
\hline $\begin{array}{c}\text { Like a Rolling } \\
\text { Stones }\end{array}$ & Evolución & Proceso & Narrativa & Paisaje \\
\hline Continuará... & Paradoja & Todo/partes & Experiencia & Cine \\
\hline
\end{tabular}

Para una mejor comprensión, a continuación se ejemplifica la sesión que lleva por título: Transparencias.

Idea vector Inimaginable: entendemos que una formación que incorpora los principios de la complejidad debe estar abierta a la indeterminación. Ello comporta asumir la imposibilidad de conocer y/o controlar la totalidad de variables que convergen en un contexto educativo y, por lo tanto, estar abierta a que aparezcan situaciones inimaginables.

Esfera conceptual techo de cristal: una formación que se aproxima a descubrir nuevas identidades y relaciones, y que asume los riesgos en este proceso de descubrimiento, se puede concretar en el concepto de techo de cristal, un límite que a menudo nos bloquea en el contexto personal o colectivo y del que no somos claramente conscientes. Es lo que separa lo que es imaginable de lo que es inimaginable.

Esfera didáctica representar: en nuestro caso se diseñan dinámicas que permitan trabajar los límites que componen el techo de cristal de forma abierta y creativa. Los participantes representan sus límites y los comparten para explorar y construir nuevas oportunidades.

Esfera creativa instalaciones: la transparencia se expresa a través de instalaciones de arte contemporáneo. Se diseña un espacio que genera itinerarios o subespacios que juegan con elementos de distintas calidades de transparencia, por ejemplo, pasillos laberínticos construidos con plástico translúcido para acceder a la sala de trabajo. Tras las instalaciones transparentes se intuyen espacios simbólicos con límites difusos donde aparece aquello que en un principio es inimaginable.

\subsection{Decisiones metodológicas}

La investigación que se presenta es de tipo evaluativo y en concreto se evalúa un programa formativo desde la perspectiva de la Ambientalización Curricular. La investigación pretende obtener información del propio programa mediante toda la muestra de profesores y educadores que participan en él y con la finalidad de 
detectar el grado de presencia de la ambientalización curricular y proponer orientaciones de mejora y continuidad. Por ello se ha optado por una investigación de carácter mixto inductivo-deductivo que permita capturar todos aquellos aspectos que expresan los participantes sin un filtro a priori que puede provocar la pérdida de datos significativos para los objetivos planteados.

El instrumento de recogida de datos ha sido la entrevista semiestructurada con bajo grado de directividad, siendo la técnica utilizada el registro audiovisual. Para ello se ha situado a cada participante en una sala con formato de plató televisivo y se le ha pedido autorización para realizar una entrevista de una duración máxima de 10 minutos en la que se ha grabado la intervención de la persona en primer plano y cámara fija. Así pues, se ha garantizado un marco de privacidad, pues únicamente se encontraban en la sala la persona encargada de la cámara, la que realizaba las preguntas y la persona entrevistada.

La entrevista semiestructurada se ha focalizado en las siguientes preguntas: (i) ¿Qué aspectos de la formación recibida destacarías en relación a la Ambientalización Curricular?; (ii) ¿Qué elementos has incorporado en tu contexto profesional en ambientalización curricular de la programación recibida?; (iii) ¿Podrías comentar otros aspectos relevantes del programa que no has expresado en las respuestas anteriores?

La estructura de la entrevista responde a un formato estándar. Una primera parte de introducción donde se han compartido los objetivos y se ha creado un clima adecuado. Una segunda parte, de carácter más estructurado, donde se ha profundizado en los aspectos clave de la entrevista, y una parte final, menos estructurada, donde las personas entrevistadas han podido expresar aquellos aspectos que consideraban relevantes y no habían aparecido anteriormente. En ningún caso se han redirigido las respuestas de los entrevistados esperando que el discurso respondiera al máximo a sus intereses e inquietudes respecto a la experiencia formativa.

Las entrevistas se han realizado durante la última sesión de trabajo del programa, de forma paralela a la realización de actividades. Durante el día, de forma individual, cada participante se ha ausentado del gran grupo para ir a una sala adyacente a realizar la entrevista. Cada participante se ha ausentado un máximo de 15 minutos.

\subsection{Análisis de los datos}

El análisis de los datos se ha realizado en clave de análisis del contenido. Para ello se ha trabajado en diversas fases:

I. Audición reiterativa de las respuestas que se dan en las entrevistas

Se han escuchado varias veces las respuestas de cada persona entrevistada destacando aquellos aspectos que resultan relevantes tanto desde un análisis global como del análisis de aspectos puntuales del discurso. 
II. Selección de segmentos de significatividad en función de su contenido

Una vez realizada la audición se han determinado aquellos segmentos de significatividad que se consideran relevantes para los objetivos de la investigación. Los diferentes segmentos se han transcrito en una tabla, seguidamente se han seleccionado conceptos clave de cada segmento que se consideran las evidencias que hacen que dicho segmento tenga el carácter de significativo (Mateo Andrés y Vidal Xifre, 2000).

\section{Conceptualización y asignación de categorías}

En una primera fase, se ha realizado el análisis de diez entrevistas y se han agrupado los conceptos clave de manera deductiva (categorías modelo Aces), definiendo las subcategorías de forma inductiva. Dichas subcategorías obedecen a la asignación de un patrón temático compartido que ha permitido dotar de marco conceptual al análisis.

En una segunda fase, se ha reiterado el proceso anterior con la totalidad de las entrevistas con el objetivo de enriquecer tanto el número de subcategorías como su conceptualización. Así, por ejemplo, las palabras: Motivación, Experiencia vivida, Superación de obstáculos, Refuerzo emocional, Optimismo, Creer en el programa, Agrado, Probar, Sorprender, Enriquecimiento, Diversión, Agotamiento cognitivo, Confianza, Bienestar, Libertad, Intenso, Intranquilidad, Seguridad, Momentos de abandonar se han agrupado en la subcategoría Emocional a la que se han asignado todos los conceptos clave que hacen referencia al estado de ánimo de los participantes respecto a la oferta del programa formativo. Dicha subcategoría se ha asociado de forma deductiva a la categoría: Considerar los aspectos cognitivos y afectivos de las personas, que propone el modelo ACES.

\section{Triangulación de datos}

En este punto se ha realizado una doble triangulación de los datos obtenidos para garantizar su validez y fiabilidad. Se han realizado dos procesos de análisis paralelos por parte de dos investigadores. El primero ha sido de carácter temporal por el que cada análisis de datos se ha desarrollado de forma completa en dos momentos separados por tres meses de diferencia. Cada una de las personas que ha realizado el análisis ha comparado los datos obtenidos detectando elementos consistentes y señalando las incoherencias. El segundo ha consistido en una triangulación de expertos donde se han comparado los resultados obtenidos por cada investigador estableciendo acuerdos por consenso y discutiendo tanto discrepancias como desajustes detectados en la triangulación temporal para obtener, finalmente, categorías y subcategorías de consenso. 
V. Obtención de resultados preliminares

El trabajo realizado ha permitido obtener tablas de análisis de cada una de las personas participantes donde se obtienen las categorías que han sido relevantes para cada persona de la muestra.

Seguidamente se han agrupado todos los datos obteniendo de este modo categorías y subcategorías del total de la muestra de profesionales que han participado en el programa. Se ha seleccionado toda la información relevante independientemente de su frecuencia en el global de los datos. De este modo se ha pretendido obtener el máximo de matices que permitan tener un conocimiento profundo del posicionamiento respecto al programa de los perfiles profesionales participantes.

\section{Resultados}

A continuación, se presentan los resultados globales correspondientes a una visión de conjunto del grupo de participantes. Los resultados obtenidos nos permiten realizar dos lecturas. Una por categorías, que nos permite profundizar en la presencia de cada una de las categorías que nos propone el modelo ACEs, y una de conjunto, que nos permite determinar el grado de ambientalización curricular de la propuesta. Los resultados se muestran siguiendo los objetivos y preguntas que orientan la investigación.

En relación al objetivo 1: Identificar los factores del programa formativo que son significativos en relación a la Ambientalización Curricular

\section{a) ¿Qué categorías de la Ambientalización Curricular destacan los participantes en el programa formativo?}

Se muestran los resultados organizados por tablas. Cada tabla corresponde a uno de las características que propone el modelo ACES.

I. Forma de mirar el mundo

\begin{tabular}{|l|l|}
\hline \multicolumn{1}{|c|}{ CONCEPTOS CLAVE } & \multicolumn{1}{c|}{ SUBCATEGORÍA } \\
\hline Nuevas visiones, Nuevas ideas & \\
Incorporación de nuevos conceptos & \\
Filosofía de la EA & Enriquecimiento de la concepción \\
Marco teórico sobre asesoramiento & \\
Conectar teoría y práctica & \\
Abstracción & \\
\hline
\end{tabular}




\begin{tabular}{|l|l|}
\hline \multicolumn{1}{|c|}{ CONCEPTOS CLAVE } & \multicolumn{1}{c|}{ SUBCATEGORÍA } \\
\hline Techo de cristal & \\
Inimaginable & IAN \\
Intangible & \\
Diversidad de perspectivas & \\
\hline Conexión, Establecer relaciones & \\
Límites & Complejidad \\
Azar & \\
Límites & \\
Bidireccionalidad & \\
Cambio/proceso & \\
\hline
\end{tabular}

Los resultados muestran que los participantes:

- Enriquecen la forma de mirar el mundo al incorporar nuevos enfoques y maneras de pensar de la educación para la sostenibilidad y, a la vez, al establecer relaciones entre teoría y práctica.

- Consideran la propuesta de Idea Vector como eje vertebrador del proceso formativo.

- Incorporan algunos de los principios de la complejidad como forma de aproximarse a los fenómenos del mundo.

II. Orden disciplinar: flexibilidad y permeabilidad

\begin{tabular}{|l|l|}
\hline \multicolumn{1}{|c|}{ CONCEPTOS CLAVE } & \multicolumn{1}{|c|}{ SUBCATEGORÍA } \\
\hline $\begin{array}{l}\text { Visión de conjunto } \\
\text { Dinámicas globales integradoras de saberes }\end{array}$ & Estructura de la propuesta \\
\hline $\begin{array}{l}\text { Interdisciplinariedad } \\
\text { Excelente nivel de formación } \\
\text { Diversidad de lenguajes }\end{array}$ & Diálogo entre saberes \\
\hline
\end{tabular}

Los resultados muestran que los participantes:

- Tienen consciencia de que la estructura de la propuesta favorece un enfoque integrador y global, para la cual se precisa relacionar las disciplinas.

- Consideran que el diálogo de saberes es una aproximación necesaria que incluye relaciones entre conocimientos y movilización de distintos lenguajes desde una formación de calidad. 


\section{Contextualización: Local-Global-Local; Global-Local-Global}

\begin{tabular}{|l|l|}
\hline \multicolumn{1}{|c|}{ CONCEPTOS CLAVE } & SUBCATEGORÍA \\
\hline Importancia del contexto & \\
Incorporación del contexto/ & Contexto \\
medio/escenario & \\
Búsqueda de nuevos escenarios & \\
para trabajar diferentes actitudes & Aplicabilidad: Elementos favorecedores \\
Aplicabilidad & \\
Innovación & \\
Marco orientador de la acción & \\
Contexto difícil & Aplicabilidad: Elementos obstaculizadores \\
Dificultad comunicación con el equipo & \\
Reflexión del equipo & \\
Poco tiempo uara realizar cambios & \\
\hline Participación de los docentes & \\
Legitimación del trabajo del profesional & \\
Investigación y práctica en el aula & \\
Cambios globales en el centro & \\
Soporte al centro educativo & Agentes implicados \\
Favorecer la implicación de las escuelas & \\
Implicación administración pública & \\
\hline $\begin{array}{l}\text { Implicación personal } \\
\text { Procesos reflexivos } \\
\text { Soporte de otros profesionales } \\
\text { De la teoría a la práctica } \\
\text { Conducir el cambio } \\
\text { Idear miniprogramas } \\
\text { Relativizar }\end{array}$ & \\
\hline
\end{tabular}

Los resultados muestran que los participantes:

- Tienen consciencia de la relevancia del contexto y de la necesidad de considerarlo desde una perspectiva amplia.

- Valoran el contexto como espacio de acción transformadora y en este sentido la presencia de elementos favorecedores y obstaculizadores de la acción.

- Consideran una visión escalar de la diversidad de agentes que forman el contexto y la necesidad de estimular cambios en todos ellos.

- Ponen en relevancia la necesidad de que los docentes sean protagonistas del cambio como agentes transformadores que asumen responsabilidades. 
IV. Tener en cuenta el sujeto en la construcción del conocimiento

\begin{tabular}{|l|l|}
\hline \multicolumn{1}{|c|}{ CONCEPTOS CLAVE } & \multicolumn{1}{|c|}{ SUBCATEGORÍA } \\
\hline Contacto con otras personas & \\
Sinergias & \\
Ampliación red & \\
Falta de convivencia & Red \\
Relaciones interpersonales & \\
Integración ideas & \\
Trabajo cooperativo & \\
Consenso en los planteamientos & \\
Difusión & \\
Proximidad & \\
Acompañamiento & Aprendizaje \\
\hline Actualización de conocimientos & \\
Abstracción & \\
Avance & \\
Reflexión intensa a nivel personal & Autorregulación \\
\hline Descubrimiento y búsqueda de nuevos retos & \\
Encontrar estrategias para dar respuesta a las & \\
necesidades y demandas & \\
Favorecer la significatividad de los programas & \\
y las actividades & Aprendizaje de recursos \\
Adquirir nuevos recursos & \\
Nuevos lenguajes & \\
Referente teórico & \\
Microrrelatos & \\
\hline Crecimiento personal & \\
Competencia creativa & \\
\hline Reflexión sobre el proceso & \\
Pensar y hacer & \\
Profundizamiento en mi propio perfil & \\
\hline
\end{tabular}

Se puede observar que los participantes destacan:

- La red como estructura comunicativa aparece como elemento clave del trabajo. La red aporta soporte emocional, riqueza en el intercambio y elaboración de nuevas ideas.

- El aprendizaje orientado hacia el enriquecimiento, la abstracción que ha implicado a los propios individuos. 
- El programa ha aportado recursos para actuar en el aula que se valoran como significativos, se redunda en que los recursos están fundamentados teóricamente.

- La capacidad de reflexión y presencia de la creatividad en la actividad profesional de los participantes.

- El protagonismo de los participantes en el aprendizaje, ya que conectan reflexión personal con experiencia formativa.

V. Considerar los aspectos cognitivos y afectivos de las personas

\begin{tabular}{|l|l|}
\hline \multicolumn{1}{|c|}{ ConCEPTOS ClAVE } & SUBCATEGORÍA \\
\hline Diversidad emocional & \\
Introducción elementos estimulantes & \\
y motivadores & \\
Interesantes: & \\
Motivación & \\
- Experiencia vivida & \\
- Superación de obstáculos & \\
- Optimismo & \\
- Creer en el programa & \\
- Agrado & \\
- Srobar & \\
- Enriquecimiento & \\
- Diversión & \\
- Agotamiento cognitivo & \\
- Confianza & \\
- Bienestar & \\
- Libertad & \\
- Intenso & \\
- Intranquilidad & \\
- Seguridad & \\
- Momentos de abandonar & \\
- Proceso substancial & \\
- Gratificante & \\
- Componente de sorpresa & \\
- Componente estimulante & \\
- Aventura & \\
- Dienestar & \\
- Coherencialtad de explicar & \\
- Refuerzo emocional & \\
- Complicidad emocional & \\
\hline
\end{tabular}


LA IDEA VECTOR Y SUS ESFERAS: UNA PROPUESTA FORMATIVA PARA LA AMBIENTALIZACIÓN CURRICULAR...

\begin{tabular}{|l|l|}
\hline \multicolumn{1}{|c|}{ CONCEPTOS CLAVE } & \multicolumn{1}{c|}{ SUBCATEGORÍA } \\
\hline $\begin{array}{l}\text {-Sorpresa en el aula } \\
\text { Seguridad }\end{array}$ & Emocional \\
\hline $\begin{array}{l}\text { Compromiso } \\
\text { Coherencia } \\
\text { Clima de aula }\end{array}$ & Valores \\
\hline
\end{tabular}

Los resultados muestran que los participantes:

- Detectan una intensa presencia de la perspectiva emocional que combina una visión positiva de la experiencia con dudas que se entienden como fases por la que pasan para obtener aprendizaje.

- Ponen en relevancia, desde la perspectiva de los valores, la creación de un clima de aula que favorece el desarrollo del programa formativo desde el compromiso y la coherencia.

VI. Adecuación metodológica: coherencia y construcción entre teoría y práctica

\begin{tabular}{|l|l|}
\hline \multicolumn{1}{|c|}{ CONCEPTOS CLAVE } & \multicolumn{1}{|c|}{ CATEGORÍa } \\
\hline $\begin{array}{l}\text { Cambio en las metodologías } \\
\text { Creatividad } \\
\text { Nuevos enfoques } \\
\text { Presentación estimulante y motivadora } \\
\text { Herramientas para el asesoramiento de } \\
\text { centros }\end{array}$ & \\
\hline $\begin{array}{l}\text { Alto nivel de capacidad de trabajo } \\
\text { Programa pequeño } \\
\text { Realismo en los objetivos } \\
\text { Marco de referencia }\end{array}$ & \\
\hline $\begin{array}{l}\text { Favorecer la incorporación de competencias } \\
\text { Relato } \\
\text { Diversidad de dinámicas } \\
\text { Pequeñas actividades }\end{array}$ & \\
\hline $\begin{array}{l}\text { Alto nivel de trabajo } \\
\text { Alto nivel de implicación } \\
\text { Complicidad } \\
\text { Dominio de diversidad de recursos } \\
\text { Cuestionamientos }\end{array}$ & Recursos formativos, nuevas metodologías \\
\hline
\end{tabular}

Se puede observar que los participantes destacan: 
- El grado de innovación del programa destacando cambios en metodologías, enfoques y la posibilidad de obtener recursos.

- La coherencia en el desarrollo del programa haciendo énfasis en el carácter posibilista de la propuesta por su dimensión de planteamiento realista.

- Las metodologías y recursos que se han aportado también se han utilizado de forma significativa y funcional a lo largo del programa.

- La competencia del equipo organizador se valora como un elemento clave del programa tanto por su conocimiento en el tema como su actitud hacia el programa.

VII. Orientación prospectiva de escenarios alternativos

\begin{tabular}{|l|l|}
\hline \multicolumn{1}{|c|}{ CONCEPTOS CLAVE } & \multicolumn{1}{c|}{ SUBCATEGORÍA } \\
\hline $\begin{array}{l}\text { Amplitud de perspectivas } \\
\text { Apertura de nuevos horizontes de la EA } \\
\text { Diversidad de aspectos y enfoques de la EA }\end{array}$ & Reconceptualización de la EA \\
\hline $\begin{array}{l}\text { Cambio global } \\
\text { Consciencia de cambios }\end{array}$ & Cambio \\
\hline Expectativas para madurar y consolidar & Continuidad \\
\hline Futuro abierto & Futuro \\
\hline
\end{tabular}

Se puede observar que los participantes destacan:

- Un alto nivel de reconceptualización de la educación para la sostenibilidad que se fundamenta en nuevas perspectivas que determinan nuevos enfoques

- El trabajo en escenarios alternativos favorece la conciencia de la necesidad de cambios, los cuales deben dar respuesta a una visión global

- La necesidad de madurar dichos cambios en un proceso personal de cambio continuo abierto a un futuro incierto.

\section{b) ¿Qué oportunidades para la Ambientalización Curricular aparecen durante el desarrollo del programa formativo?}

En referencia a las oportunidades un elemento clave es el estatus de liderazgo que se otorga al equipo que ha dirigido el programa. En este sentido parece clave un estilo de liderazgo soportado en el conocimiento respecto al tema, una alta capacidad de trabajo y la apertura al cambio en función de la respuesta de los participantes. Ello puede generar la confianza que permita a los participantes adentrarse en un proceso innovador que a menudo genera inquietud. 
Los participantes destacan como la complejidad y las IAN, que han sido los ejes teóricos que han orientado el programa, constituyen elementos clave para reflexionar sobre la propia concepción de Educación para la Sostenibilidad e incluso enriquecerla.

El grado de estructuración del programa y la aplicabilidad de los contenidos trabajados se destacan como aspectos clave para favorecer la consciencia entre los participantes de que la propuesta es transferible al aula.

Se destaca como elemento clave que el trabajo realizado está fundamentado, lo que comporta asumir la necesidad de no caer en el activismo y dotarse de un marco teórico que permita orientar las decisiones.

Se observa que el trabajo en red, como estructura organizativa, se constituye como un elemento clave para que los participantes se sientan parte de una red de soporte que les estimule a innovar en el aula y reflexionar sobre el proceso llevado a cabo.

Por último, destacar que la intensidad emocional con la que se ha vivido el proceso constituye para los participantes un elemento clave para valorar positivamente la formación. En este sentido destacar que no se trata de una emoción por la emoción, fácilmente provocadora y vacía de contenido. Se trata de descubrir la emoción que provoca el aprendizaje, la duda, el saberse expuesto a la novedad y a la vez gozar del desequilibrio y reequilibrio cognitivo.

\section{c) ¿Qué obstáculos para la Ambientalización Curricular aparecen durante el desarrollo del programa formativo?}

Se destacan como obstáculos relevantes la necesidad de tener un amplio bagaje cultural para llevar a cabo propuestas como las que se han mostrado a lo largo del programa. Ello comporta la necesidad de hacer conscientes a los participantes de su conocimiento como profesionales de la educación y como educadores ambientales y entender que la construcción del propio bagaje cultural es un proceso continuo que requiere esfuerzo y reporta satisfacción.

Un segundo obstáculo constituye la concepción de que el contexto profesional no favorece su aplicación de las propuestas presentadas a lo largo del programa, tanto en referencia a los equipos docentes como a las instituciones. En este sentido aparece un tema recurrente en la formación de educadores ambientales que se concreta en la sensación de ir a contracorriente. Parece relevante la necesidad de reflexionar sobre estrategias de intervención en el propio contexto que permitan implementar nuevas propuestas que, sin perder de vista las limitaciones del contexto, sean capaces de detectar y aprovechar sus oportunidades.

En definitiva se destaca la participación en un programa que plantea una apuesta clara por el cambio, lo que constituye en sí mismo una oportunidad y un bloqueo. Oportunidad porque abre nuevas perspectivas y plantea nuevas posibilidades. Obstáculo porque apostar por el cambio implica asumir riesgos e 
inseguridades que para ser superados exigen adoptar la condición de expertos que no se ha consolidado en los participantes y crear espacios de confianza.

En relación al objetivo 2: Proponer orientaciones de continuidad del programa formativo en relación a la mejora de la Ambientalización Curricular. Para ello nos preguntamos:

¿Qué orientaciones de continuidad se derivan de los resultados de la investigación?

Cuando hacemos referencia a las perspectivas de continuidad de un programa de Ambientalización Curricular como el que se presenta aparecen dos ejes fundamentales: la transferencia del conocimiento adquirido a otros contextos y la estructura que ha de tomar el grupo para continuar con el proceso formativo.

Entendemos por transferencia de conocimiento aquella acción que realizan los participantes en el programa que posibilita impactar con los conocimientos adquiridos en un nuevo contexto favoreciendo procesos de transformación. La transferencia se puede entender como un proceso escalar donde se visualizan las escalas: personal, grupal, contexto profesional y el propio contexto donde se enmarca el programa.

A nivel personal el proceso de transferencia implica que cada uno de los participantes ha de buscar situaciones en que pueda mostrarse competente para diseñar, implementar y evaluar experiencias de Ambientalización Curricular como las que ha desarrollado a lo largo del programa. Ello implica desarrollar una actitud fundamentada en la acción innovadora, la búsqueda de información, el desarrollo de materiales curriculares y entornos estimulantes para la educación para la sostenibilidad que constituyen vectores para favorecer la ambientalización del currículum desde la acción individual.

Paralelamente la red establecida con los participantes del programa constituye un valor fundamental en el momento de desarrollar las actitudes anteriores. Por ello se hace necesario establecer una red estable de colaboraciones, complicidades y de "amigos críticos» que permita que la acción individual vaya acompañada del refuerzo entre iguales, que se encuentran en procesos similares en diversidad de centros educativos. El valor del grupo ya no es el de espacio de intercambio sino el de contexto de soporte, impulsor y regulador de la calidad de la propia acción didáctica.

Más allá de lo personal y grupal, la Ambientalización Curricular toma sentido en el marco de una institución educativa por lo que la siguiente escala sobre la que actuar es el contexto profesional, centro educativo o colectivo asesor. En este sentido las personas participantes en el programa pueden desarrollar un liderazgo derivado del carácter de expertos que han ido desarrollando a lo largo de su formación. La transferencia eficaz no se da por imperativo ni por imitación, sino que necesita personas que orienten el proceso, que generen confianza en las personas del entorno y que actúen como líderes cuando la situación lo requiere. Se hace pues fundamental que los participantes en el curso aprovechen su bagaje para, 
en el triángulo hacer, pensar y comunicar, impulsar la comunicación fluida en su entorno como punto de inicio de procesos de acción innovadora.

Pensamos que los resultados obtenidos y la finalidad del programa presentan la necesidad de hacer llegar el trabajo realizado a las diferentes escalas del territorio y redes del ámbito. Ello implica un cambio de rol de los participantes en el programa, que dejan de ser discentes, para pasar a convertirse en agentes activos en los diversos programas, es decir, elementos distorsionadores que pueden enviar fluctuaciones al resto de la red con la finalidad de impulsar su cambio, en una u otra dirección.

\section{CONClusiones}

El desarrollo del programa ha permitido obtener algunas claves para orientar el desarrollo de un proceso formativo en Ambientalización Curricular coincidente con los principios ACES y con impacto real en las personas que participan en la formación. Una formación que evidencia la vinculación entre formación e investigación en el campo de la educación para la sostenibilidad para descubrir y definir nuevos patrones (Calafell y Bonil, 2014). A modo de conclusiones pensamos que dichas claves se pueden resumir en cuatro: credibilidad, cosmovisión, conceptualización y acción. Desarrollemos brevemente cada una de ellas.

El programa debe ser creíble para los participantes. Ello comporta un intenso trabajo para los organizadores que va más allá de preparar sesiones interesantes. Comporta que en lo explícito y lo implícito los participantes reciben evidencias de que el trabajo propuesto está sostenido intelectualmente. Se puede considerar que un programa de innovación se mueve entre el escepticismo y la militancia, especialmente cuando toma la innovación como eje estructurador, por ello es fundamental la exigencia del propio equipo formativo en la preparación de las sesiones (materiales, ritmo de trabajo, calidad de las actividades propuestas, fundamentación teórica, coherencia teórico-práctica...). También es fundamental que los participantes reconozcan al equipo formador como un equipo de expertos, más allá de las personas individuales, lo que exige una gran coherencia entre diversidad individual y coherencia colectiva. Al mismo tiempo los formadores deben mostrar su liderazgo de expertos, lo que implica no renunciar a los retos que aparecen, justificar sólidamente todos los juicios y asumir los puntos débiles de la propuesta como retos a abordar.

El programa debe ofrecer a los participantes una nueva cosmovisión. A menudo en educación para la sostenibilidad se habla de la necesidad de generar nuevas cosmovisiones en el alumnado, pero al mismo tiempo se hace fundamental que la propia educación para la sostenibilidad constituya un ejemplo de nueva cosmovisión. Por ello el programa debe ser capaz de proponer nuevas reglas, nuevas claves formativas. En el caso que nos ocupa elementos como las Ideas Vectores o las esferas han constituido elementos innovadores para los participantes y la clave para construir una nueva cosmovisión de la educación para la sostenibilidad. 
Aparece por lo tanto un nuevo reto cuando se pone en evidencia que una nueva cosmovisión del mundo se soporta en una nueva cosmovisión de la educación para la sostenibilidad. Asumir este silogismo implica aceptar el riesgo, el azar, la necesidad de innovar de forma permanente, la apertura a la crítica y la necesidad de construir de forma permanente como los ejes sobre los que se elabora una propuesta formativa. En el caso de nuestro programa dicha cosmovisión se mueve en el eje simple-complejo e intenta que a lo largo de la formación los participantes descubran los principios de la complejidad, tengan criterios para decidir si los quieren utilizar y orientaciones para aplicarlos en contextos concretos.

El programa debe favorecer la reflexión sobre el concepto de Ambientalización Curricular de los participantes. Todas las personas que se dedican a la educación para la sostenibilidad tienen una concepción de la Ambientalización Curricular, sea explícita o implícita, una concepción que no siempre plantea coherencias entre lo que se piensa y la forma en que se actúa. Cuando nos planteamos un programa que quiere impactar sobre la concepción de Ambientalización Curricular de los participantes se hace fundamental planificar espacios en que cada persona tenga la oportunidad de contrastar lo que piensa con las nuevas ideas y pueda decidir si reconfigura sus modelos iniciales. En el programa formativo este proceso se ha movido en el eje confirmación/reformulación. Algunos individuos muestran que el programa les ha confirmado sus ideas iniciales, pues la forma en que las han incorporado a sus esquemas mentales les ha permitido fundamentarlas más si cabe. Otros participantes han realizado procesos de reformulación que han llegado a lo más profundo de las concepciones de ambientalización curricular de los participantes. Parece óptimo que cada individuo atrape las ideas que el programa ha ofrecido y las incorpore a sus esquemas mentales de forma no neutral, sino transformándolas y haciendo el esfuerzo de hacerlas útiles, bien dentro de la concepción que tenía, o bien haciendo el esfuerzo de configurar un nuevo esquema mental.

El programa se debe orientar a capacitar para la acción transformadora. Como hemos comentado el trabajo realizado tiene sentido en la medida que capacita a los participantes a actuar en su entorno estimulando el cambio. No sirve únicamente plantear un cambio orientado a la sostenibilidad, sino que la Ambientalización Curricular ha de transformarse a sí misma para constituirse como un sistema sostenible. Aparece de este modo una forma de redundancia que exige que la Ambientalización Curricular esté ambientalizada en sí misma. De este modo la acción se mueve en el eje reflexión/intervención. A lo largo del programa se ha fomentado la reflexión y son los participantes, en su entorno, los que deben favorecer la intervención. Pero no sirve cualquier acción, es necesaria una acción fundamentada en la reflexión, que huya del activismo para convertirse en una estrategia fundamentada. Una forma de intervenir que fundamenta la acción y favorece que más allá de la intervención se convierta en una forma de transformar la cultura institucional.

En definitiva, podemos afirmar que el modelo formativo propuesto ha tenido impacto diverso en los participantes. Se ha mostrado como un modelo con una estructura clara, que muestra coherencia entre los elementos que lo forman y 
establece conexiones claras entre ellos, lo que permite redundar en su carácter de credibilidad. Es capaz de poner el foco en la muestra de nuevas cosmovisiones y dar claves para construirlas en forma de ideas vector, lo que nos acerca a su gran finalidad. Es un modelo que presenta elementos de anclaje en los esquemas mentales de los participantes, conectando con sus concepciones de educación para la sostenibilidad, desequilibrándolas y estimulando que aparezcan nuevas, lo que lo convierte en un modelo significativo para los participantes. El grado de adopción de los elementos del modelo se distribuye según el conocimiento de los participantes, que muestran el mayor grado en referencia a la esfera didáctica y el menor en referencia a la Idea Vector, lo que convierte la apropiación de la propuesta formativa en un proceso continuo en el que es conveniente que aparezcan todas las esferas. Por último, es un modelo formativo que muestra un alto potencial transformador del contexto, dicha transformación tiene como condición necesaria pero no suficiente que los equipos educativos decidan transformarse a sí mismos, una decisión que ha de tomar cada participante.

\section{REFERENCIAS BIBLIOGRÁFICAS}

Bauman, Z. (2007) Vida de consumo. Madrid, Fondo de Cultura Económica.

BECK, U. (1997). La sociedad del riesgo, hacia una nueva modernidad. Barcelona, Paidós.

Bonil, J.; Calafell, G.; Granados, J.; JunYent, M. y Tarín, R. M. (2012) Un modelo formativo para avanzar en la Ambientalización Curricular. Revista de Formación de Profesorado, 16 (2), $145-163$.

Bonil, J.; Calafell, G.; Márquez, C. y Pujol, R. M. (2004). La integración del paradigma de la complejidad a la formación científica como vía de acceso a la Ambientalización Curricular: las preguntas mediadoras y el diálogo disciplinar, en GELI, A. M. y JUNYENT, M. (eds.) Ambientalización Curricular de los Estudios Superiores. Girona, Universitat de Girona - Red ACES, 187-208.

Bonil, J.; Calafell, G.; Orellana, L.; Espinet, M. y Pujol, R. M. (2004) El diálogo disciplinar, un camino necesario para avanzar hacia la complejidad. Investigación en la Escuela 53, 83-97. Sevilla: Diada.

Bonil, J.; Junyent, M. y Pujol, R. M. (2010) Educación para la sostenibilidad desde la perspectiva de la complejidad. Revista Eureka de Enseñanza y Divulgación de las Ciencias, 7 (n. ${ }^{\circ}$ extraordinario), 198-215.

Bonil, J. y PUjol, R. M. (2008) El paradigma de la complejidad, un marco de referencia para el diseño de un instrumento de evaluación de programas en la formación inicial de profesorado. Enseñanza de las Ciencias, 26 (1), 5-22.

Bonil, J. y Pujol, R. M. (2011) Educación científica a propósito de la palabra crisis. Enseñanza de las Ciencias, 29 (2), 251-262.

Bonil, J.; SANMARTí, N.; Tomàs, C. y Pujol, R. M. (2004) Un nuevo marco para dar respuesta a las dinámicas sociales: el paradigma de la complejidad. Investigación en la Escuela, 53, 5-19. Sevilla: Díada.

CADEP (2005) Directrices para la sostenibilización curricular - CRUE. Documento aprobado por el Comité Ejecutivo del Grupo de Trabajo de Calidad Ambiental y Desarrollo Sostenible de la CRUE. Valladolid, 18 de abril de 2005. 
Calafell, G. (2010) L'emergència del diàleg disciplinari com a oportunitat per incorporar la complexitat en l'educació científica (Tesis Doctoral). Universitat Autònoma de Barcelona.

CALAfELl, G. Y BonIL, J. (2014) Identificación y caracterización de las concepciones de medio ambiente de un grupo de profesionales de la educación ambiental. Enseñanza de las Ciencias, 32 (3), 205-225.

CAlafell, G.; JunYent, M. y Bonil, J. (2015) Una propuesta para ambientalizar el currículum. Cuadernos de Pedagogía, 460, 56-60.

CANElo, J.; JunYent, M. y Bonil, J. (2015) Innovación y creatividad para favorecer un pensamiento sistémico-crítico: ideas de alto nivel en la formación inicial de maestros. Foro de Educación, 13 (19), 125-140. doi: http://dx.doi.org/10.14516/fde.2015.013.019.006.

Davis, B. y Sumara, D. (2007) Complexity Science and Education: Reconceptualizing the Teacher's Role in Learning. Interchange, 38 (1), 53-67.

Geli, A. M. (2002) Universidad, sostenibilidad y Ambientalización Curricular, en ARBAT, E. y Geli, A. M. (eds.) Ambientalización curricular de los Estudios Superiores: Aspectos Ambientales de las Universidades. Girona: Servei de Publicacions Universitat de Girona /Red ACES.

Geli, A. M.; Junyent, M. y Arbat, E. (2003) El cambio hacia la sostenibilidad de los estudios de la Universidad de Girona, en Junyent, M.; Geli, A. M. y Arbat, E. (eds.) Proceso de caracterización de la Ambientalización Curricular de los estudios superiores. Girona: Servei de Publicacions Universitat de Girona/Red ACES.

Geli, A. M.; Junyent, M. y SÁnchez, S. (eds.) (2004) Diágnóstico de la Ambientalización Curricular de los Estudios Superiores. Girona: Servei de Publicacions Universitat de Girona/Red ACES.

GIL, J. (1994) Análisis de datos cualitativos. Aplicaciones a la investigación educativa. Barcelona, Promociones Publicaciones Universitarias (PPU).

GUNi - Global University NeTWORK FOR INNOVATION (2012) Higher Education in the World 4. Higher Education Commitment's to Sustainability: from Understanding to Action. Londres: Palgrave.

IzQuierdo, M.; Espinet, M.; Bonil, J. y Pujol, R. M. (2004) Ciencia escolar y complejidad. Investigación en la Escuela, 53, 21-29.

Junyent, M.; Geli, A. M. y Arbat, E. (2003) Características de la Ambientalización Curricular: Modelo aces, en Junyent, M.; Geli, A. M. y ARBAt, E. (eds.) Proceso de caracterización de la Ambientalización Curricular de los estudios superiores. Girona, Servei de Publicacions Universitat de Girona/Red ACES.

LOVElock, J. (2007) La venjança de la Terra. Per què es revolta la Terra i com encara podem salvar la bumanitat. Barcelona, Columna.

Mateo Andrés, J. y Vidal Xifre, M. C. (2000) Mètodes d'investigació. Barcelona: Universitat Oberta de Catalunya.

Mayer, M. (2002) Ciudadanos del barrio y del planeta, en Imbernon, F. (ed.) Cinco ciudadanías para una nueva educación. Barcelona, Graó, 83-104.

MAYOR, F. (2009) La problemática de la sostenibilidad en un mundo globalizado. Revista de Educación, número extra (1), 25-52.

Pujol, R. M. (2000) Ambientalització i escola. Perspectiva Escolar, 235, 2-7.

PuJOL, R. M. y Bonil, J. (2003) Una propuesta de Ambientalización Curricular desde la formación científica: el caso del crecimiento humano, en JunYent, M.; GeLI, A. M. y ARBAT, 
LA IDEA VECTOR Y SUS ESFERAS: UNA PROPUESTA FORMATIVA PARA LA AMBIENTALIZACIÓN CURRICULAR...

E. (eds.) Proceso de caracterización de la Ambientalización Curricular de los estudios superiores. Girona, Servei de Publicacions Universitat de Girona/Red ACES.

SAUvÉ, L. (2006) La educación ambiental y la globalización: Desafíos curriculares y pedagógicos. Revista Iberoamericana de Educación, 41, 83-101.

Tilbury, D.; Podger, P. y ReID, A. (2004) Action research for Change Towards Sustainability: Change in Curricula and Graduate Skills Towards Sustainability, Final Report prepared for the Department of the Environment and Heritage and Macquarie University.

Tilbury, D. y Wortman, D. (2004) Engaging people in Sustainability. IUCN Publications Services Unit, Commission on Education and Communication. 